Pacific Journal of Mathematics

RADICAL SUBCATEGORIES 


\section{RADICAL SUBCATEGORIES}

\section{MARY GRAY}

In a categorical settiug we generalize the concept of radical as defined for groups and for rings. We define semiabelian and co-semi abelian categories. Such categories lack the convenient additive structure of the sets of morphisms between two objects, which may be derived from the duality of the axioms for abelian categories, but, for example, the concept of semi-abelian categories permits one to consider the categories of abelian groups, all groups, commutative rings with identity all rings, rings with minimum condition, Lie algebras and compact Hausdorff spaces with base points and continuous maps under the same categorical formulation. Generalizations of the classical radical properties are proved; for example, the fact that any object in a semi-abelian category is the extension of a radical object by a semi-simple object and the dual statement.

As special cases of our radical subcategory, we have Jacobson-, Levitzki-, McCoy-radicals, the Lie algebra radical, the Plotkin radical and the torsion subgroup of an abelian group; an example of a coradical subcategory is found in topological groups.

Maranda [6] has also defined a radical using category terminology, but only in the category of all unitary right modules over some ring $R$, while Dickson [1] has considered a similar notion in Abelian categories.

II. Semi-abelianess. A category $\mathscr{C}$ with a zero-object is semiabelian if

(1) every morphism of $\mathscr{C}$ may be factored into its coimage followed by its image and

(2) every morphism has a cokernel and co-semi abelian if (1) holds and

(2*) every morphism has a kernel. Note that abelian categories are both semi-abelian and co-semi abelian.

EXAMPLES. 1. The category of all rings and ring homomorphisms is semi-abelian and co-semi abelian and has as product the direct product.

2. The subcategory of 1 whose objects are commutative rings with identity and morphisms the ring homomorphisms between them is semi-abelian and co-semi abelian. The tensor product is the coproduct. 
3. The subcategory of 1 whose objects are rings with minimum conditions and ring homomorphisms between them is semi-abelian.

4. The category of Lie algebras and Lie algebra homomorphisms is semi-abelian and co-semi abelian with the direct product as product.

5. The category of all groups and group homomorphisms is semiabelian and co-semi abelian with the direct product as product and the free product as coproduct.

6. The subcategory of 5 consisting of finite groups and group homomorphisms is semi-abelian and co-semi abelian with products. It does not have coproducts; for suppose $X$ were the coproduct of $J_{2}$ and $J_{2}$. Consider the maps $f_{n}: J_{2} \rightarrow G_{n}, g_{n}: J_{2} \rightarrow G_{n}$ where (1) $f=a \neq$ identity $\neq b=(1) g$ and $G_{n}$ is defined by the relations $a \neq b, a^{2}=b^{2}=$ identity, $(a b)^{n}=$ identity. Since the union of the images of $f_{n}$ and $g_{n}$ generates $G$, any map from the coproduct to $G_{n}$ which has the desired properties must be an epimorphism. Hence the coproduct has arbitrarily large homomorphic images and must be infinite.

7. The category of compact Hausdorff spaces with base points and continuous maps (taking base point into base point) is semi-abelian and co-semi abelian with products and coproducts. For the product, we use the ordinary Cartesian product, and for the coproduct we first take the disjoint union of two spaces; then the quotient space resulting from the identification of base points in the disjoint union is the coproduct of the two spaces.

We need the following properties of categories.

Proposition 2.1. If $A \rightarrow B$ is a kernel, then kernel (cokernel $(A \rightarrow B))=A \rightarrow B$ (if cokernel $(A \rightarrow B)$ exists).

Proof. Let $A \rightarrow B$ be the kernel of $B \rightarrow C, B \rightarrow D$ the cokernel of $A \rightarrow B$. Let $X \rightarrow B$ be such that $X \rightarrow B \rightarrow D=0$. We have

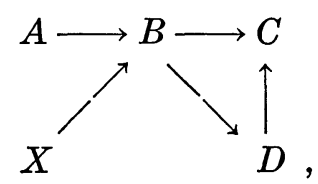

where the triangle commutes.

Hence $X \rightarrow B \rightarrow C=0$; thus there exists a morphism $X \rightarrow A$ such that

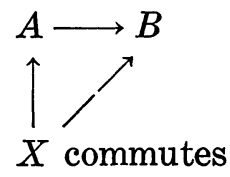

and $A \rightarrow B$ is the kernel of $B \rightarrow D$.

And dually 
Proposition 2.1*. If $A \rightarrow B$ is a cokernel, then cokernel (kernel $(A \rightarrow B))=A \rightarrow B$ (if the kernel exists).

Proposition 2.2. Let $\mathscr{C}$ be a semi-abelian or co-semi abelian category. If two subobjects of $\mathscr{C}$, represented by $A_{1} \rightarrow A$ and $A_{2} \rightarrow A$, $A_{1} \rightarrow A$ a kernel of $A \rightarrow F$, have a g. l. b., its domain is the domain of the kernel of the morphism $A_{2} \rightarrow A \rightarrow F$. Conversely, if $A_{1} \rightarrow A$ and $A_{2} \rightarrow A$ represent subobjects, $A_{1} \rightarrow A$ a kernel of $A \rightarrow F$, then if the kernel of $A_{2} \rightarrow A \rightarrow F$ exists, it is the g.l. b. of $A_{1} \rightarrow A$ and $A_{2} \rightarrow A$.

Proof. Suppose $A_{12} \rightarrow A$ is the g. l. b. of $A_{1} \rightarrow A$ and $A_{2} \rightarrow A$. Then $A_{12} \rightarrow A_{2} \rightarrow A \rightarrow F=0$. Suppose $X \rightarrow A_{2} \rightarrow F=0$. Let $I \rightarrow A_{2}$ be the image of $X \rightarrow A_{2}$. Since $I \rightarrow A \rightarrow F=0$ and $A_{1} \rightarrow A$ is the kernel of $A \rightarrow F$, there exists a morphism $I \rightarrow A_{1}$ such that

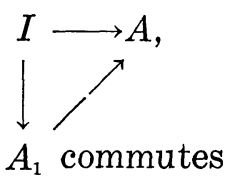

i. e., the subobject represented by $\mathrm{I} \rightarrow A_{1}$ is contained in that represented by $A_{1} \rightarrow A$. Since we already have its containment in $A_{2} \rightarrow A$, it is contained in the g.l.b. Thus there is a morphism $I \rightarrow A_{12}$ such that

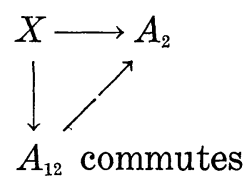

and $A_{12} \rightarrow A_{2}$ is the kernel.

On the other hand, let $A_{12} \rightarrow A_{2}=\operatorname{kernel}\left(A_{2} \rightarrow A \rightarrow F\right)$. We observe that $A_{12} \rightarrow A_{2} \rightarrow A \rightarrow F=0$ and since $A_{1} \rightarrow A=\operatorname{kernel}(A \rightarrow F)$ there is a morphism $A_{12} \rightarrow A_{1}$ such that

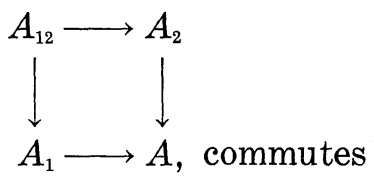

Hence $A_{12} \rightarrow A$ is a lower bound. Suppose $X \rightarrow A$ is contained in $A_{1} \rightarrow A$ and $A_{2} \rightarrow A$. Then since $X \rightarrow A_{2} \rightarrow F=X \rightarrow A_{1} \rightarrow F=0$ and $A_{12} \rightarrow A_{2}=\operatorname{kernel}\left(A_{2} \rightarrow F\right)$, there is, by definition of kernel, a unique morphism $X \rightarrow A_{12}$ such that $X \rightarrow A_{12} \rightarrow A_{2}=X \rightarrow A_{2}$. Moreover, $X \rightarrow A_{12} \rightarrow A_{1}=X \rightarrow A_{1}$ since $X \rightarrow A_{12} \rightarrow A_{1} \rightarrow A=X \rightarrow A_{1} \rightarrow A$ and $A_{1} \rightarrow A$ is right cancellable. Hence $X \rightarrow A_{i}$ is contained in $A_{12} \rightarrow A_{i}$, $i=1,2$. 
Proposition 2.2*. Let $\mathscr{C}$ be a semi-abelian or co-semi abelian category. If two quotient objects in $\mathscr{C}$, represented by $A \rightarrow A_{1}$ and $A \rightarrow A_{2}, A \rightarrow A_{1}$ a cokernel of $K \rightarrow A$, have a g.l.b., its range is the range of the cokernel of the morphism $K \rightarrow A \rightarrow A_{2}$. Conversely if $A \rightarrow A_{1}$ and $A \rightarrow A_{2}$ represent subobjects and $A \rightarrow A_{1}$ is a cokernel of $K \rightarrow A$, then if the cokernel of $K \rightarrow A \rightarrow A_{2}$ exists, it is the g.l.b. of $A \rightarrow A_{1}$ and $A \rightarrow A_{2}$

Proposition 2.3. If we consider the assignments of kernels and cokernels as functions on the set of (representatives of) quotient objects and on the set of (representatives of) subobjects of an object, respectively, then they are order-reversing where defined.

Proof. Suppose $A \rightarrow B_{1}, A \rightarrow B_{2}$ are epimorphisms and

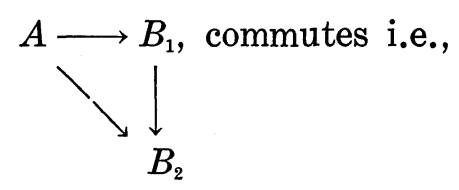

$A \rightarrow B_{1}$ contains $A \rightarrow B_{2}$. Let $K_{i} \rightarrow A$ be the kernel of $A \rightarrow B_{i}$. Then $K_{1} \rightarrow A \rightarrow B_{2}=0$ since

$$
K_{1} \rightarrow A \rightarrow B_{2}=K_{1} \rightarrow A \rightarrow B_{1} \rightarrow B_{2}=K_{1} \rightarrow B_{1} \rightarrow B_{2}=0 .
$$

Hence there exists $K_{1} \rightarrow K_{2}$ such that

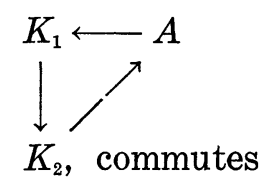

The proof is dual if we suppose containment of monomorphisms.

The sequence

$$
0 \rightarrow A \rightarrow B \rightarrow C \rightarrow 0
$$

is exact if $A \rightarrow B$ is a kernel of $B \rightarrow C$ and $B \rightarrow C$ is a cokernel of $A \rightarrow B$.

PROPOSITION 2.4. (for a category with coproducts) In the sequence

$$
0 \longrightarrow A \stackrel{u_{1}}{\longrightarrow} A+B \stackrel{\left(\begin{array}{l}
0 \\
1
\end{array}\right)}{\longrightarrow} B \longrightarrow 0
$$

$A \stackrel{u_{1}}{\longrightarrow} A+B$ is a monomorphism and $A+B \stackrel{\left(\begin{array}{l}0 \\ 1\end{array}\right)}{\longrightarrow} B$ is the cokernel of $u_{1}$, where the $u_{i}, i=1,2$, are defining morphisms for the coproduct 
of $A$ and $B$.

Proof. $u_{1}$ is a monomorphism since $u_{1}\left(\begin{array}{l}1 \\ 0\end{array}\right)$ is. To prove that $\left(\begin{array}{l}0 \\ 1\end{array}\right)$ is a cokernel of $u_{1}$, let $A+B \stackrel{h}{\longrightarrow} X$ be a morphism such that $u_{1} h=0$. Then we can write $h$ as $\left(\begin{array}{l}u_{1} h \\ u_{2} h\end{array}\right)$. Then $u_{1} h=0$ and $\left(\begin{array}{l}u_{1} h \\ u_{2} h\end{array}\right)=\left(\begin{array}{l}0 \\ 1\end{array}\right) u_{2} h$ since $u_{2}\left(\begin{array}{l}0 \\ 1\end{array}\right) u_{2} h=u_{2}\left(\begin{array}{l}u_{1} h \\ u_{2} h\end{array}\right)$ and by definition of coproduct the map from $A+B$ must be unique.

REMARK. The sequence is exact if all monomorphisms are kernels, since, by Proposition 2.1, $u_{1}$ is the kernel of $\left(\begin{array}{l}0 \\ 1\end{array}\right)$. However, in the case of groups a component group is not necessarily a normal subgroup of the free product, e.g., $J$ in $J+J$, so in semi-abelian categories the sequence may fail to be exact.

Proposition 2.4*. (for a category with products) In the sequence

$$
0 \longrightarrow A \stackrel{(1.0)}{\longrightarrow} A \times B \stackrel{p_{2}}{\longrightarrow} B \longrightarrow 0
$$

$A \stackrel{(0,1)}{\longrightarrow} A \times B$ is the kernel of $p_{2}$ and $A \times B \stackrel{p_{2}}{\longrightarrow} B$ is an epimorphism, where $p_{i}, i=1,2$, are the defining morphisms for the product of $A$ and $B$.

REMARK. By duality and Proposition 2.1*, the sequence is exact if all epimorphisms are cokernels. In general $A \times B \stackrel{p_{2}}{\longrightarrow} B$ may fail to be a cokernel. For consider the product of the unit interval with itself in the category of Example 7 above.

Suppose $p_{1}: I_{1} \times I_{2} \rightarrow I_{1}$ is the cokernel of $f: X \rightarrow I_{1} \times I_{2}$. Since $I_{2} \rightarrow I_{1} \times I_{2}$ is the kernel of $p_{1}, X f$ is contained in $I_{2}$. Hence we retract the unit square into the triangle $\{(x, y) \mid y \leqq x\}$ by a map $r$ such that the complement of the triangle collapses onto the diagonal and the triangle remains fixed. Then there is no map from $I_{1}$ to the triangle which composed with $p_{1}$ gives the retraction $r$.

Proposition 2.5. If $A \times B \stackrel{p_{1}}{\longrightarrow} A$ or $A \times B \stackrel{p_{2}}{\longrightarrow} B$ is a cokernel, their g.l.b. is the zero-morphism.

Proof. By Proposition 2.3* the g.l.b. is the zero-morphism, for cokernel $(A \rightarrow A \times B \rightarrow A)=\operatorname{cokernel}\left(A \stackrel{{ }^{1} A}{\longrightarrow} A\right)=0$. 
Proposition 2.5*. If $A \stackrel{u^{1}}{\longrightarrow} A+B$ or $B \stackrel{u_{2}}{\longrightarrow} A+B$ is a kernel, their g.l.b. is the zero-morphism.

III. Radical and co-radical subcategories. Suppose $\mathscr{C}$ is a semiabelian category and $\mathscr{R}$ a full subcategory of $\mathscr{C}$. We call $\mathscr{R}$ a radical subcategory of $\mathscr{C}$ if the following axioms are satisfied;

(R1) If $A \in \mathscr{R}$, then if $f \in \operatorname{hom}(A, B)$ for some $B \in \mathscr{C}$, domain image $(A \rightarrow B) \in \mathscr{R}$. If $S \rightarrow A$ is a kernel, $S \in \mathscr{R}$.

(R2) For each $G \in \mathscr{C}$ there exists a unique (up to equivalence) morphism which is the l.u.b. in the set of all subobjects of $G$ which are kernels and whose domains are objects of $\mathscr{R}$. We denote this morphism by $x_{G}$ and call it the radical of $G$.

(R3) The radical of range cokernel $x_{a}$ is the zero-morphism.

If $\mathscr{C}$ is a co-semi abelian category with a full subcategory $\mathscr{R}^{*}$ which satisfies the following axioms, $\mathscr{R}^{*}$ is called a co-radical subcategory:

(R1*) If $B \in \mathscr{R}^{*}$, then if $f \in \operatorname{hom}(A, B)$ for some $A \in \mathscr{C}$, range coimage $f \in \mathscr{R}^{*}$ and if $B \rightarrow C$ is a cokernel, $C \in \mathscr{R}^{*}$.

(R2*) For each $G \in \mathscr{C}$, there exists a unique morphism which is the l.u.b. in the set of all quotient objects of $G$ which are cokernels and whose ranges are objects of $\mathscr{R}^{*}$. We denote this morphism by $X_{G *}$ and call it the co-radical of $G$.

$\left(\mathrm{R} 3^{*}\right)$ The co-radical of domain kernel $x_{\theta}{ }^{*}$ is the zero-morphism.

The objects of the radical subcategory are called radical objects. We call a subcategory $\mathscr{S}$ of a semi-abelian category $\mathscr{C}$ a semi-simple subcategory with respect to a radical subcategory $\mathscr{R}$ if $S \in \mathscr{S}$ implies that the radical of $S$ is the zero-morphism. Dually, we define coradical objects and co-semi-simple subcategory.

EXAMPLES. 1. Plotkin [5] has given the following characterization of radical groups:

Suppose $\beta$ is a property of groups satisfying

(1) Every homomorphic image of a $\beta$-group is a $\beta$-group. Every normal subgroup of a $\beta$-group is a $\beta$-group.

(2) Every group $G$ has a normal $\beta$-subgroup which contains all other normal $\beta$-subgroups of $G$. We denote this subgroup by $\beta(G)$.

Then we form a $\beta$-series

$$
1=\beta_{0}(G) \subseteq \beta_{1}(G) \subseteq \cdots \leqq \beta_{\alpha}(G) \subseteq \beta_{\alpha+1}(G) \subseteq \cdots
$$

as follows:

(1) If $\alpha$ is a limit ordinal $\beta_{\alpha}(G)=\mathrm{U}_{\gamma<\alpha} \beta_{\gamma}(G)$.

(2) $\beta_{\alpha+1}(G) / \beta_{\alpha}(G)=\beta\left(G / \beta_{\alpha}(G)\right)$. 
If $\gamma$ is the first ordinal for which $\beta_{\gamma}(G)=\beta_{\gamma+1}(G)$, we call $\beta_{\gamma}(G)=\bar{\beta}(G)$ the $\beta$-radical of $G$. If $\bar{\beta}(G)=G, G$ is a $\beta$-radical group.

The radical groups (with respect to some property $\beta$ ) form a radical subcategory of the category of groups, for:

(R1) This is a consequence of the following result of Plotkin [5]:

If a group $G$ has an ascending normal series all of whose factor groups are $\beta$-groups, $G$ is $\beta$-radical.

For if $G$ has such a series, we can form series for normal subgroups and factor groups (and products in the group-theoretic sense). (Our radical is the inclusion map of this radical into the group.)

(R2) and (R3) $\bar{\beta}(G)$ exists for every group $G$, is clearly itself radical and $G / \bar{\beta}(G)$ must be semi-simple from its construction and if $\bar{\beta}(G)$ were strictly contained in another $\beta$-radical normal subgroup this would not be the case. On the other hand, if $R$ is a $\beta$-radical normal subgroup it is contained in $\bar{\beta}(G)$, for by the remark above, its product with $\bar{\beta}(G)$ is radical.

Consider the nil or Hirsch-Plotkin "radical," the maximum locally nilpotent normal subgroup of a group. The groups which have no non-trivial homomorphic image with trivial Hirsch-Plotkin radical form a radical subcategory, for local nilpotency is a property such as described in the characterization of Plotkin.

2. The classes of Jacobson-, Levitzki-, McCoy-semi-simple rings are semi-simple subcategories of the category of rings and ring homomorphisms. We show, as an example, that Jacobson radical rings form a radical subcategory using the quasi-regular characterization. The proofs in the other cases are similar.

(R1) Any ideal or quotient ring of a quasi-regular ring is clearly quasi-regular.

(R2) The Jacobson radical is defined in any ring to be the sum of the quasi-regular ideals, which is itself quasi-regular, and hence it is the l.u.b. in the subcategory of quasi-regular rings. The radical is as above the inclusion map of this ideal into the ring.

(R3) If $\bar{z}$ is an element of the radical of $\mathrm{R} / J$, where $J$ is the Jacobson radical of $\mathrm{R}$, and $z$ is its pre-image in $\mathrm{R}, z r$ is quasi-regular for all $r$ in $R$, so $z$ is in the Jacobson radical of $R$, i.e., $\bar{z}=0$.

A necessary condition for a property of ideals to determine a radical subcategory is for the sum of radical objects to be a radical object. For example, the property of being quasi-regular or of being semi-nilpotent determines a radical subcategory of the category of rings but that of being nilpotent does not (the sum of nilpotent ideals is not in general nilpotent). However, in the semi-abelian category of rings with minimum condition the property of nilpotency does determine a radical subcategory. Indeed, the radicals mentioned above 
coincide in this case with the classical or nilpotent radical.

3 . The radical of a non associative algebra. That the maximum solvable ideal of a Lie algebra determines a radical subcategory of the category of (finite-dimensional) Lie algebras and Lie algebra homomorphisms is easily shown (cf., e.g., Jacobson [3]). Moreover, by the correspondence between Lie algebras and (simply-connected) Lie groups we get that a radical subcategory of the category of Lie groups is determimined by the maximum solvable subgroup of a Lie group. Similarly, the maximum nilpotent ideal of a Jordan or alternative algebra determines a radical subcategory.

4. The co-radical. We consider the dual category to the category of abelian groups. By the Pontrjagin duality theorems, there is a contravariant functor with a contravariant inverse from the category of abelian groups to the category of compact abelian (Hausdorff) topological groups which assigns to each group its character group. Hence, we consider a co-radical which is dual to the torsion subgroup of an abelian group. In the category of abelian groups, the torsion groups constitute a radical subcategory, for:

(R1) Clearly, any subgroup or factor group of a torsion group is a torsion group.

(R2) By definition, the inclusion map of the torsion subgroup of an abelian group into the group itself is maximal with respect to the subcategory of torsion groups.

(R3) If $T$ is the torsion subgroup of an abelian group $A$, then $A / \mathrm{T}$ must be torsion-free.

It is the case that the character group of a torsion group is totally disconnected and that the character group of a torsion-free group is connected (cf. Pontrjagin [6], p. 148). Thus the totally disconnected groups form a co-radical subcategory of the category of compact abelian topological groups. The co-radical is defined for any group in this category by taking the factor group with the component of the identity.

IV. Properties of radicals and co-radicals. An extension of an object $A$ by an object $B$ is an exact sequence

$$
0 \longrightarrow A \longrightarrow C \longrightarrow B \longrightarrow 0 \text {. }
$$

We call $C$ an extension object.

Proposition 4.1. The zero-oject is the only object which is both radical and semi-simple with respect to a radical subcategory $\mathscr{R}$ of a semi-abelian category.

Proof. Suppose $R \in \mathscr{R}$ and $0 \rightarrow R=$ radical $R$. Then 0 is the 
largest subobject of $\mathrm{R}$ in $\mathscr{R}$. Hence $R=0$.

Proposition 4.1*. The zero-object is the only object which is both co-radical and co-semi-simple with respect to a co-radical subcategory of a co-semi abelian category.

THEOREM 4.2. Every object of a semi-abelian category $\mathscr{C}$ is an extension object of a radical object by a semi-simple object (with respect to any radical subcategory of $\mathscr{C}$.)

Proof. For $G \in \mathscr{C}$, let $x_{G}: A \rightarrow G$ be the radical of $G$ with respect to a radical subcategory of $\mathscr{C} ; G \rightarrow B$ the cokernel of $x_{G} \cdot x_{G}$ is a kernel, so kernel $(G \rightarrow B)=x_{\theta}$, i.e., $0 \rightarrow A \rightarrow G \rightarrow B \rightarrow 0$ is exact with $A$ radical and $B$ semi-simple.

THEOREM 4.2*. Every object of a co-semi abelian category $\mathscr{C}^{*}$ is an extension object of a co-semi-simple object by a co-radical object.

THEOREM 4.3. If $x$ is the radical of $A, S \rightarrow A$ a kernel and $K \rightarrow S$ the radical of $S$, then if $K \rightarrow A$ is a kernel, it is equivalent to the g.l.b. of $x$ and $S \rightarrow A$ (if it exists).

Proof. Let $K_{1} \rightarrow A$ be the g.l.b. of $x$ and $S \rightarrow A$. Then $K_{1} \rightarrow$ domain $x$ is a kernel by Proposition 2.3 so $K_{1} \in \mathscr{R}$. Hence $K_{1} \rightarrow S$ is contained in $K \rightarrow S$. On the other hand, $K \rightarrow A$ is a kernel so it is contained in $x$ by (R2), as well as in $S \rightarrow A$ and hence in their g.l.b.

TheOREM 4.3*. If $x$ is the co-radical of $A, A \rightarrow B$ a cokernel, and $B \rightarrow C$ the co-radical of $B$, then if $A \rightarrow C$ is a cokernel, it is equivalent to the g.l.b. of $x$ and $A \rightarrow B$ (if it exists).

THeOREM 4.4. A radical subcategory $\mathscr{R}$ of a semi-abelian category $\mathscr{C}$ with products such that $A_{1} \times A_{2} \stackrel{p^{i}}{\longrightarrow} A_{i}$, is a cokernel in $\mathscr{C}$ for $i=1$ or 2 for each pair of objects $A_{1}, A_{2} \in \mathscr{R}$ is semi-abelian with products.

Proof. Note that under these hypotheses $A_{1} \times A_{2} \rightarrow A_{1}$ is the cokernel of $A_{2} \stackrel{(0,1)}{\longrightarrow} A_{1} \times A_{2}$ or $A_{1} \times A_{2} \rightarrow A_{2}$ is the cokernel of

$$
A_{1} \stackrel{(1,0)}{\longrightarrow} A_{1} \times A_{2},
$$

since by Proposition $2.4^{*}, A_{i} \rightarrow A_{1} \times A_{2}$ is the kernel of

$$
A_{1} \times A_{2} \rightarrow A_{j}, i \neq j,
$$


and by Proposition 2.1* a cokernel is the cokernel of its kernel.

Let $A_{1}, A_{2} \in \mathscr{R}, A_{1} \times A_{2}$ be their product in $\mathscr{C}$. Then the g.l.b. of $A_{1} \times A_{2} \rightarrow A_{1}$ and $A_{1} \times A_{2} \rightarrow A_{2}$ is the zero-morphism. By Proposition 2.3, the l.u.b. of $A_{1} \rightarrow A_{1} \times A_{2}$ and $A_{2} \rightarrow A_{1} \times A_{2}$ is $A_{1} \times A_{2} \rightarrow A_{1} \times A_{2}$. Hence by (R2), $A_{1} \times A_{2} \in \mathscr{R}$.

The semi-abelianess follows from (R1), with the existence of a zero-object being clear.

THEOREM 4.4*. A co-radical subcategory $\mathscr{R}$ of a co-semi abelian category $\mathscr{C}$ with coproducts such that $A_{i} \stackrel{u_{i}}{\longrightarrow} A_{1}+A_{2}$ is a kernel for $i=1$ or 2 for each pair of objects $A_{1}, A_{2} \in \mathscr{R}$, is co-semi abelian with coproducts.

Note that a category with a zero-object, factorization and products of arbitrary sets of objects is semi-abelian, and dually coproducts of arbitrary sets of objects give the existence of kernels [3].

THeOREM 4.5. If $\mathscr{R}_{1}$ and $\mathscr{R}_{2}$ are radical subcategories of a semiabelian category $\mathscr{C}$ and $x_{G}^{i}$ is the $\mathscr{R}_{i}$ radical of $G \in \mathscr{C}$, then $\mathscr{R}_{1}$ is a subcategory of $\mathscr{R}_{2}$ if and only if $x_{G}^{1}$ is contained in $x_{G}^{2}$ for each $G \in \mathscr{C}$.

Proof. Suppose $\mathscr{R}_{1}$ is a subcategory of $\mathscr{R}_{2}$. Then domain $x_{G}^{1} \in \mathscr{R}_{2}$ and hence $x_{G}^{1}$ is contained in $x_{G}^{2}$ by (R2).

Suppose $x_{G}^{1}$ is contained in $x_{G}^{2}$ for all $G \in \mathscr{C}$. Let $R \in \mathscr{R}_{1}$. Then $1_{R}$ is contained in $x_{R}^{2}$ and hence they are equivalent, i.e., $R \in \mathscr{R}_{2}$.

THEOREM 4.5*. If $\mathscr{R}_{1}$ and $\mathscr{R}_{2}$ are co-radical subcategories of a co-semi abelian category $\mathscr{C}$ and $x_{G}^{i}$ is the $\mathscr{R}_{i}$ co-radical of $G \in \mathscr{C}$, then $\mathscr{R}_{1}$ is a subcategory of $\mathscr{R}_{2}$ if and only if $x_{G}^{1}$ is contained in $x_{G}^{2}$ for each $G \in \mathscr{C}$.

We define a simple object to be an object $A$ such that $X \rightarrow A$ a kernel implies that $X=A$ or $X=0$. Then if none of the simple objects of a semi-abelian category is a radical object, the product of simple objects is semi-simple.

In a certain type of semi-abelian category one can define abelian objects [3]; then it is possible to select an abelian subobject of each object in the category so that the objects selected form an abelian subcategory satisfying (R1). However, in general the l.u.b. of abelian subobjects of a given object is not abelian, nor is it the case that the range of the cokernel of the l.u.b. is necessarily "semi-simple." For 
example, in the category $\mathscr{G}$ of groups and group homomorphisms consider the quaternion group of order eight. It has three subgroups of order four which are abelian but whose l.u.b. is the entire (non-abelian) group. Hence the abelian groups do not form a radical subcategory of $\mathscr{G}$.

Moreover, any radical subcategory of $\mathscr{G}$ whose objects are all abelian must be the trivial subcategory (i.e., all of its objects are trivial groups), for suppose $\{0\} \neq G \in \mathscr{R}$, a radical subcategory of $\mathscr{G}$ all of whose objects are abelian. Then by (R1) $J_{p} \in \mathscr{R}$ for some prime $p$. $\mathscr{R}$ does not contain all $p$-proups, for there exist non-abelian finite $p$-groups. Let $X$ be a minimal finite $p$-group not contained in $\mathscr{R}$ and $\mathrm{R}(X)$ its radical. Then $[X: \mathrm{R}(X))]=p$ by minimality of $X$. But $X / R(X)=J_{p} \in \mathscr{R}$, i.e., $X / R(X)$ is not semi-simple.

\section{REFERENCES}

1. S. E. Dickson, A torsion theory for Abelian categories, Trans. Amer. Math. Soc. 121 (1966), 223-235.

2. Peter Freyd, Abelian Categories. Harper and Row, New York, 1964.

3. Mary Gray, Abelian objects, Pacific. J. Math. (to appear)

4. Nathan Jacobson, Lie Algebras, John Wiley and Sons, New York, 1962.

5. Saunders MacLane, Categorical algebra, Bull. Amer. Math. Soc. 71 (1965). 40-107.

6. J.-M. Maranda, Injective structures, Trans. Amer. Math. Soc. 110 (1964), 98-135.

7. B. I. Plotkin, Radical groups, Mat. Sbornik, N. S. (79) 37 (1955), 507-526.

8. L. Pontrjagin, Topological Groups, Princeton University Press, Princeton, N. J., 1939.

Received January 6, 1965.

UNIVERSITY OF CALIFORNIA, BERKELEY

California State College, Hayward 



\section{PACIFIC JOURNAL OF MATHEMATICS}

\section{EDITORS}

\section{H. ROYDEN}

Stanford University

Stanford, California

J. P. JANS

University of Washington

Seattle, Washington 98105

\section{J. DUGUNDJI}

Department of Mathematics

Rice University

Houston, Texas 77001

RICHARD ARENS

University of California

Los Angeles, California 90024

ASSOCIATE EDITORS

E. F. BECKENBACH

B. H. NeumanN

F. WoLF

K. YosidA

\section{SUPPORTING INSTITUTIONS}

\author{
UNIVERSITY OF BRITISH COLUMBIA \\ CALIFORNIA INSTITUTE OF TECHNOLOGY \\ UNIVERSITY OF CALIFORNIA \\ MONTANA STATE UNIVERSITY \\ UNIVERSITY OF NEVADA \\ NEW MEXICO STATE UNIVERSITY \\ OREGON STATE UNIVERSITY \\ UNIVERSITY OF OREGON \\ OSAKA UNIVERSITY \\ UNIVERSITY OF SOUTHERN CALIFORNIA
}

\author{
STANFORD UNIVERSITY \\ UNIVERSITY OF TOKYO \\ UNIVERSITY OF UTAH \\ WASHINGTON STATE UNIVERSITY \\ UNIVERSITY OF WASHINGTON \\ AMERICAN MATHEMATICAL SOCIETY \\ CHEVRON RESEARCH CORPORATION \\ TRW SYSTEMS \\ NAVAL ORDNANCE TEST STATION
}




\section{Pacific Journal of Mathematics}

\section{Vol. 23, No. 1 \\ March, 1967}

M. J. C. Baker, A spherical Helly-type theorem ................... 1

Robert Morgan Brooks, On locally m-convex*-algebras.............. 5

Lindsay Nathan Childs and Frank Rimi DeMeyer, On automorphisms of separable algebras ...................................

Charles L. Fefferman, A Radon-Nikodym theorem for finitely additive set

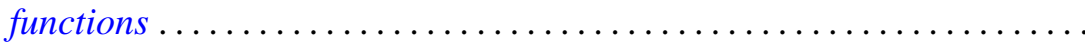

Magnus Giertz, On generalized elements with respect to linear

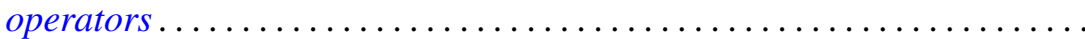

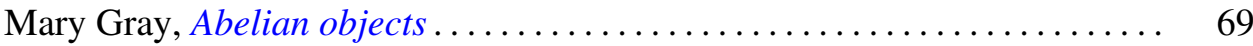

Mary Gray, Radical subcategories.............................. 79

John A. Hildebrant, On uniquely divisible semigroups on the two-cell . . . . . 91

Barry E. Johnson, AW*-algebras are $\mathrm{QW}^{*}$-algebras ............... 97

Carl W. Kohls, Decomposition spectra of rings of continuous functions . . . . 101

Calvin T. Long, Addition theorems for sets of integers .............. 107

Ralph David McWilliams, On $w^{*}$-sequential convergence and quasi-reflexivity ................................... 113

Alfred Richard Mitchell and Roger W. Mitchell, Disjoint basic

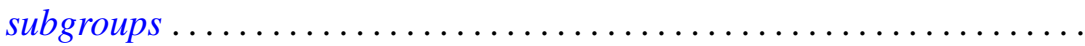

John Emanuel de Pillis, Linear transformations which preserve hermitian and positive semidefinite operators .

Qazi Ibadur Rahman and Q. G. Mohammad, Remarks on Schwarz's lemma

Neal Jules Rothman, An $L^{1}$ algebra for certain locally compact topological semigroups ...

F. Dennis Sentilles, Kernel representations of operators and their adjoints ...

D. R. Smart, Fixed points in a class of sets

K. Srinivasacharyulu, Topology of some Kähler manifolds

Francis C.Y. Tang, On uniqueness of generalized direct decompositions .

171 Albert Chapman Vosburg, On the relationship between Hausdorff dimension and metric dimension . 